ARTICLE

https://doi.org/10.1038/s41467-021-26046-9

\title{
Protein identification by nanopore peptide profiling
}

\author{
Florian Leonardus Rudolfus Lucas (10) 1,3, Roderick Corstiaan Abraham Versloot ${ }^{1,3}$, Liubov Yakovlieva ${ }^{2}$, \\ Marthe T. C. Walvoort $\mathbb{D}^{2} \&$ Giovanni Maglia (1) ${ }^{1 \times}$
}

Nanopores are single-molecule sensors used in nucleic acid analysis, whereas their applicability towards full protein identification has yet to be demonstrated. Here, we show that an engineered Fragaceatoxin $C$ nanopore is capable of identifying individual proteins by measuring peptide spectra that are produced from hydrolyzed proteins. Using model proteins, we show that the spectra resulting from nanopore experiments and mass spectrometry share similar profiles, hence allowing protein fingerprinting. The intensity of individual peaks provides information on the concentration of individual peptides, indicating that this approach is quantitative. Our work shows the potential of a low-cost, portable nanopore-based analyzer for protein identification.

\footnotetext{
${ }^{1}$ Groningen Biomolecular Sciences and Biotechnology Institute, University of Groningen, Groningen, Netherlands. ${ }^{2}$ Stratingh Institute for Chemistry, University of Groningen, Groningen, Netherlands. ${ }^{3}$ These authors contributed equally: Florian Leonardus Rudolfus Lucas, Roderick Corstiaan Abraham Versloot. $凶_{\text {email: g.maglia@rug.nl }}$
} 
T he ever-increasing demand for high-throughput proteomic studies and personalized medicine requires the development of scalable and low-cost protein analysers ${ }^{1-3}$. Modern proteomics relies heavily on tandem mass spectrometry (MS) for its high precision and capability to identify and quantify proteins in complex mixtures ${ }^{3-5}$. However, most mass analyzers are large, have a high cost of investment, are expensive to maintain, and require specialized operators to function ${ }^{2,3,6}$.

In contrast to mass spectrometry devices, nanopore-based analyzers provide a low-cost and high-throughput platform with the adaptability towards native environments ${ }^{7-10}$. In nanopore analysis, analytes are measured as they disrupt an ionic current passing through individual nanopores under an applied potential. Importantly, the magnitude of the current blockade $\left(\mathrm{I}_{\mathrm{B}}\right)$ is mainly, altought not exclusively ${ }^{11}$, proportional to the volume the analyte excludes, allowing size discrimination of chemically similar (bio)polymers such as PEG chains, DNA, proteins, and peptides ${ }^{12-24}$. Furthermore, nanopores are capable of accurately detecting a variety of molecules, including proteins and DNA, with high precision at the single-molecule level ${ }^{17,21,24-28}$. Peptides are of special interest for protein characterization, as they allow identification analogous to bottom-up MS-based proteomics.

Three types of Fragaceatoxin $\mathrm{C}(\mathrm{FraC})$ nanopores FraC-T1, FraC-T2, and FraC-T3, most likely corresponding to octameric, heptameric and hexameric pores, respectively, can be used in peptide nanopore analysis ${ }^{29}$. FraC nanopores have been shown to differentiate peptides with a resolution of $\sim 40 \mathrm{Da}^{29}$, while also enabling the detection of small chemical modifications ${ }^{30}$. Recently we have shown that at acidic $\mathrm{pH}$ values (less than $\mathrm{pH}$ $4.5)^{23}$ peptides are captured by the nanopore despite their composition, and they are most efficiently analysed at the exact $\mathrm{pH}$ of $3.8^{29}$, the condition under which the nanopore has no significant electroosmotic flow ${ }^{16}$. Furthermore, we showed that the introduction of an aromatic residue in the sensing region of $\mathrm{FraC}$ (G13F-FraC containing a glycine to phenylalanine substitution at position 13) augmented the residence time of peptides inside the nanopore and their capture efficiency ${ }^{16}$.

In this contribution, we show that G13F-FraC-T1 can be used to directly sample proteins that are digested by a protease. The resulting collected peptides describe a spectrum that can then be used to identify proteins (Fig. 1). A similar strategy relying heavily on the fingerprinting of (tryptic) peptides ${ }^{5,22}$ has been used in the early days of mass spectrometry for the detection and identification of proteins ${ }^{31-33}$. The nanopore approach might then provide a low-cost and high-throughput approach to protein identification. Furthermore, if the protease is implemented directly above the nanopore ${ }^{34}$, this approach is amenable for single-molecule identification.

\section{Results}

Calibration of the G13F-FraC-T1 nanopore. In order to characterize G13F-FraC-T1 for protein analysis, we first measured seven (synthetic) peptides (Fig. 2a) with a mass between 500 and $1700 \mathrm{Da}$ that would have resulted from the complete, in-silico, hydrolysis of Gallus-gallus lysozyme. We included a reduction/ alkylation procedure using dithiothreitol (DTT) and iodoacetamide (IAA) prior to nanopore analysis, to prevent disulfide bond formation interfering with the protein hydrolysis. The peptide signals were measured in $1 \mathrm{M} \mathrm{KCl}$ at $\mathrm{pH} 3.8$ under an applied potential of $-70 \mathrm{mV}$ and recorded at $50 \mathrm{kHz}$ filtered to $10 \mathrm{kHz}$ using an analog Bessel-filter, and further processed using a digital Gaussian filter at $5 \mathrm{kHz}$ (Fig. 2b). Under these conditions the peptides are expected to have one positive charge as the acidic residues should be mainly protonated at low $\mathrm{pH}$. Notably, this is a recurring feature for trypsinated substrates, because trypsin cleaves preferentially after a lysine or arginine residue, thus most peptides will have a positive charge next to the $\mathrm{C}$-terminus of the peptide, yielding an overall net charge of +1 . Under these conditions, numerous peptide translocation events were observed, each with a specific current blockade $\left(\mathrm{I}_{\mathrm{B}}\right)$ (Fig. 2b). For each blockade, the percentage excluded current $\left(\mathrm{I}_{\mathrm{ex}} \%\right)$ was calculated from the decrease in current observed during a blockade $\left(\Delta \mathrm{I}_{\mathrm{B}}\right)$ relative to the observed current of the open pore $\left(\mathrm{I}_{\mathrm{O}}, \mathrm{Fig}\right.$. 1$)$. We show the dwell time and excluded current as well as a histogram of the excluded current (excluded current spectrum, or $\mathrm{I}_{\mathrm{ex}} \%$ spectrum) of all seven synthetic peptides added cumulatively in equimolar concentrations (Fig. 2b). The signals corresponding to the individual peptides were further confirmed by assessing the peptides individually. Work with alpha-hemolysin, aerolysin, and FraC nanopores revealed that the relationship between the $\mathrm{I}_{\mathrm{ex}} \%$ and mass of the analyte might be complex. Although the electrical signal relates primarily on the volume excluded by the analytes ${ }^{29,35}$, other factors such as the structure of the peptide, or the interactions of the peptides with the pore surface, or with the electrolyte and other buffer elements might play a role $17,20,29,30,36-42$. In a prior contribution, utilizing a wild type

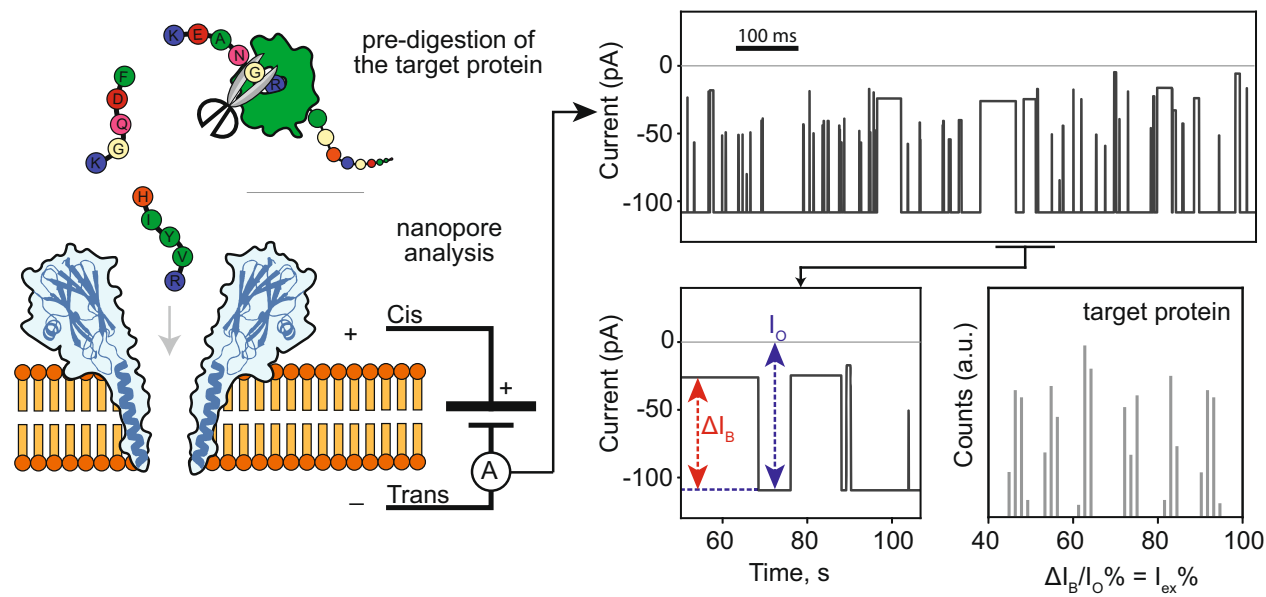

Fig. 1 Graphical overview of the nanopore protein fingerprinting approach. Peptides are pre-hydrolyzed by a specific protease (e.g. trypsin) and the resulting peptides are measured as they translocate the nanopore. Each peptide entering the nanopore reduces the open pore current $\left(I_{0}\right)$ to the blocked pore current $\left(I_{B}\right)$. The resulting excluded current $\left(\Delta I_{B}=I_{O}-I_{B}\right)$ relates to the volume of the peptide. The subsequent histogram of the percent of excluded currents $\left(\mathrm{l}_{\mathrm{ex}} \%=\Delta \mathrm{I}_{\mathrm{B}} / \mathrm{I}_{\mathrm{O}} \%\right)$ is used to identify the protein. 
a

Synthetic Tryptic Lysozyme Peptides

\begin{tabular}{|c|l|l|}
\hline Annotation & Mass (Da) & \multicolumn{1}{|c|}{ Sequence } \\
\hline 1 & 517.3 & TPGSR \\
\hline 2 & 893.4 & C(+57.02)ELAAAMK \\
\hline 3 & 874.4 & HGLDNYR \\
\hline 4 & 993.4 & WWC(+57.02)NDGR \\
\hline 5 & 1046 & GTDVQAWIR \\
\hline 6 & 1325.6 & GYSLGNWVC +57.02$)$ AAK \\
\hline 7 & 1429 & FESNFNTQATNR \\
\hline
\end{tabular}

C

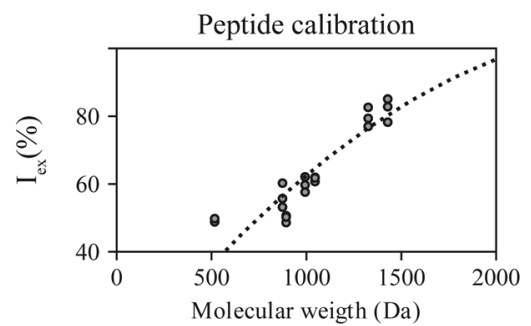

d

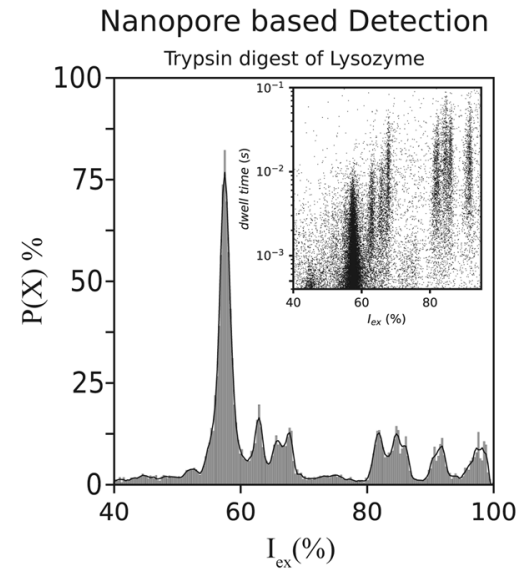

b
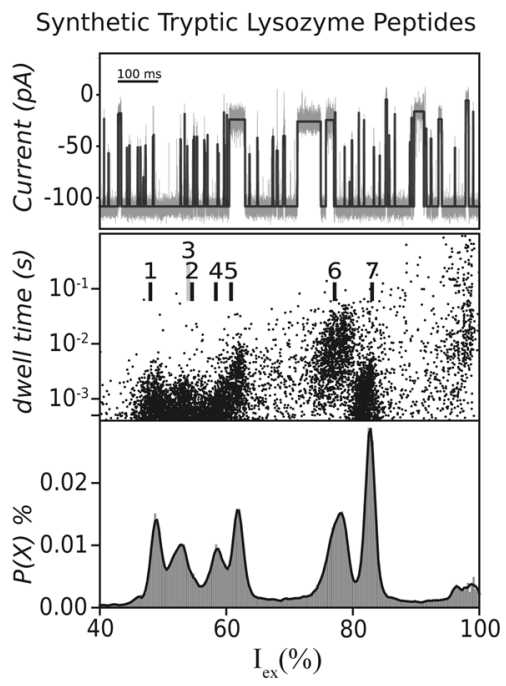

e

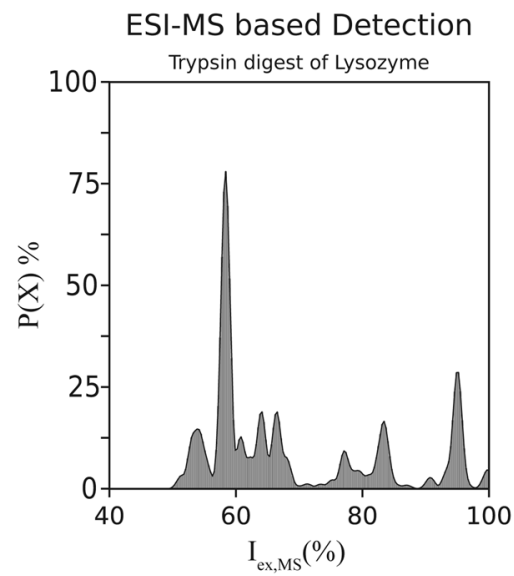

Fig. 2 Lysozyme fingerprinting using FraC-G13F-T1 nanopores. a Peptides expected from lysozyme's tryptic digest including their mass. The additional mass of 57.02 Da is expected for alkylated peptides at a cysteine position. b lonic current versus time (top), lex (\%) versus dwell time (middle) and probability density (bottom) versus $\mathrm{I}_{\mathrm{ex}}(\%)$ as obtained from the measurement of an equimolar mixture of the peptides expected from Gallus-gallus lysozyme. The black lines in the top panel indicate the fitted event. c Mass of model peptides from tryptic Gallus-gallus lysozyme (gray spheres) set against the measured excluded current (\%), based on 3 individual measurements. The black dotted line represents a polynomial fit through the data. $\mathbf{d}$ Excluded current spectrum density from the tryptic digest of Gallus-gallus lysozyme. The inset shows the dwell time versus $I_{\mathrm{ex}}(\%)$ spectrum. e Constructed $I_{\mathrm{ex}} \%$ spectrum density from the same tryptic digest of Gallus-gallus lysozyme analysed by ESI-MS. Each peak indicates a peptide identified by ESI-MS, with $\mathrm{I}_{\mathrm{ex}} \%$ calculated using the calibrated exclusion current $\%$ in $\mathbf{b}$. The height of the peak reflects the relative abundance of peptides measured by ESI-MS. The spread of the peak indicates an arbitrary standard deviation of $0.5 \mathrm{I}$ ex $\%$. All nanopore measurements were performed in $1 \mathrm{M} \mathrm{KCl}$ buffered to $\mathrm{pH} 3.8$ using $50 \mathrm{mM}$ citric acid titrated with bis-tris-propane under an applied potential of $-70 \mathrm{mV}$. Recording was performed at $50 \mathrm{kHz}$ using an analog Bessel-filter at $10 \mathrm{kHz}$ and a digital Gaussian filter of $5 \mathrm{kHz}$. Source data are provided as a Source Data file.

FraC nanopore, we described the relationship between the blockade and the mass of the peptides as a second order polynomial ${ }^{29}$. Here we also found that a second order polynomial allowed a reasonable fit (Fig. 2c), although an alternative fitting is possible (Supplementary Fig. 1) ${ }^{38}$. A better relationship will probably be obtained once the volume rather than the mass of the peptide will be accounted for.

Detection of tryptic digested Gallus-gallus lysozyme. Next, we performed the experimental tryptic digest of Gallus-gallus lysozyme, and constructed an $\mathrm{I}_{\mathrm{ex}} \%$ spectrum. The sample was alkylated and reduced using dithiothreitol/iodoacetamide, and digested using mass spectrometry-grade trypsin (Fig. 2d). We noticed that the smaller peptides, TPGSR $(1), \mathrm{C}(+57.02)$ ELAAMK (2), and HGLDNYR (3) were not clearly observed in the tryptic digest when measured using a nanopore. Electrospray ionization mass spectrometry (ESI-MS) confirmed that (1) and
(2) were not present in the sample and we could therefore assume that this is a result of incomplete cleavage. Peptide (3) is detected less efficiently by the nanopore system compared to other peptides. Possibly, this is caused by the increased charge density caused by the presence of a histidine residue in the peptide sequence, which brings an additional positive charge (at $\mathrm{pH}$ 3.8) compared to other trypsinated peptides. In turn, for small peptides this might reduce the retention inside the nanopore by a stronger electrophoretic force.

In order to compare mass spectrometry and nanopore analysis, we converted the ESI-MS measurement into an expected $\mathrm{I}_{\text {ex,Ms}} \%$ spectrum using an ad-hoc algorithm (Fig. 2e). Each peptide detected by ESI-MS was converted into a peak in the $\mathrm{I}_{\mathrm{ex}, \mathrm{MS}} \%$ spectrum, of which the specific position on the $\mathrm{x}$-axis was extrapolated from the $\mathrm{I}_{\mathrm{ex}} \%$ calibration curve from the individual lysozyme peptides (Fig. 2c). The spread (width) of the peak was introduced using an arbitrary standard deviation $(\sigma)$ of $0.5 \mathrm{I}_{\mathrm{ex}} \%$, 
while the height of the peak was derived from the relative peptide abundance from the ESI-MS measurement. Notably, we observed a good correlation between the nanopore measurements and ESIMS-based mapping (Fig. 2e). As in the case of ESI-MS, it is expected that both positively charged and hydrophobic peptides are better captured by the nanopore than peptides lacking chemical functionalities. This is because the electrophoretic migration is augmented in positively charged peptides, while the retention of hydrophobic peptides is increased by the interaction with the hydrophobic inner surface of the G13FFraC-T1 nanopore.

Protein profiling using G13F-FraC-T1. To further test the approach of protein fingerprinting using nanopores, we selected nine additional proteins with a molecular weight between 12.4 and $66.5 \mathrm{kDa}$ : cytochrome $\mathrm{C}(12.4 \mathrm{kDa})$, elongation factor P (EF$\mathrm{P}, \quad 21.0 \mathrm{kDa})^{43}$, dihydrofolate reductase (DHFR, $\left.19.1 \mathrm{kDa}\right)^{44}$, alpha casein $(24.5 \mathrm{kDa})$, beta casein $(25.1 \mathrm{kDa})$, bovine trypsin (trypsin, $23.3 \mathrm{kDa}$ ), C-terminal part of the high molecular weight adhesin 1 (HMW1ct, $34.6 \mathrm{kDa})^{45}$, proteasome-activating nucleotidase (PAN, $49.6 \mathrm{kDa}$ ), and bovine serum albumin (BSA, $66.5 \mathrm{kDa})$. For each protein, we prepared a tryptic digest and subjected the resulting peptide mixture to measurement with the FraC nanopore. From these experiments, we constructed an $\mathrm{I}_{\mathrm{ex}} \%$ spectrum (Fig. 3a, Supplementary Figs. 2, 3, and 4) and related to the expected $\mathrm{I}_{\mathrm{ex}, \mathrm{MS}} \%$ built from ESI-MS (Supplementary Fig. 5) as well as the in-silico predicted peptide masses (Supplementary Fig. 6). Rewardingly, we find that the reproducibility of the spectra is very high (Supplementary Fig. 7). Comparison between the $\mathrm{I}_{\mathrm{ex}} \%$ spectrum and the $I_{\text {ex,Ms }} \%$ revealed that the predicted $I_{\text {ex,Ms }} \%$ of
BSA, DHFR, EF-P, PAN, trypsin, and lysozyme correlated well with the experimental nanopore data, while other proteins such as cytochrome $\mathrm{C}$ and alpha casein showed a less accurate prediction displaying more peaks than observed in the nanopore. Finally, beta-casein and HMW1ct, were poorly resolved in the nanopore. We noticed that not all the peaks overlapped between the $\mathrm{I}_{\mathrm{ex}} \%$ and $\mathrm{I}_{\mathrm{ex}, \mathrm{MS}} \%$, most likely because the $\mathrm{I}_{\mathrm{ex}, \mathrm{MS}} \%$ spectra are produced considering the mass of the peptides, while current blockades relate to the volume of the analyte. Hence, nanopore analysis might require further improvements, for example by using nanopores with higher resolution or by reducing the sample complexity using chromatography devices upstream of the nanopore measurements, as is already the case in LC-MS analysis. In order to test whether nanopores are capable of distinguishing each protein, we analysed the $\mathrm{I}_{\mathrm{ex}} \%$ spectra using a spectral matching algorithm (see methods). Interestingly, we observed that all nine proteins and lysozyme are correctly assigned under these conditions (Fig. 3b), revealing that the nanopore approach can be used to identify proteins.

\section{Discussion}

Proteins are commonly identified using bottom-up mass spectrometry (MS), where proteins are enzymatically digested at specific sites and the resulting peptides are identified ${ }^{46}$. MS analysis has revolutionized our understanding of proteins and almost single handedly initiated the emerging field of proteomics $^{3-5}$. Although MS can now identify thousands of proteins in mixtures, some peptides escape MS detection and a relatively large amount of material is required. Therefore, addressing post translational modifications where only a few a
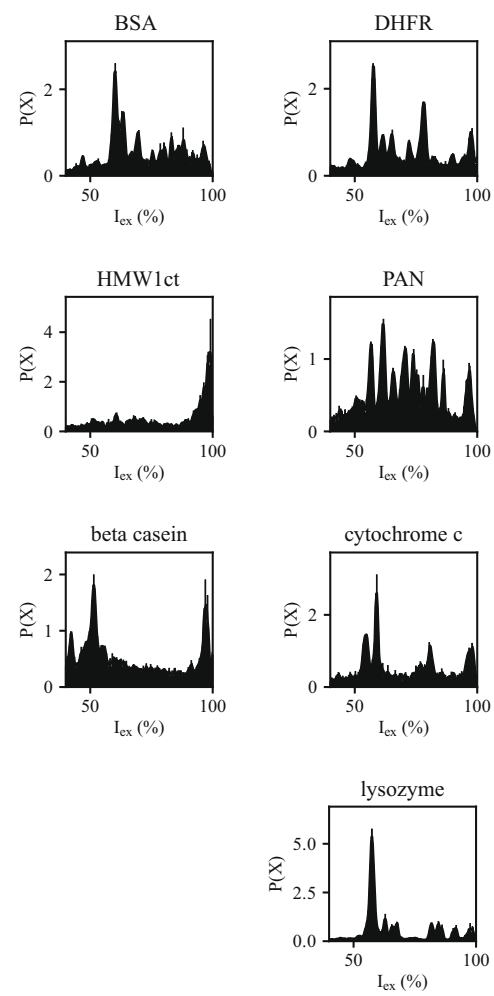

b
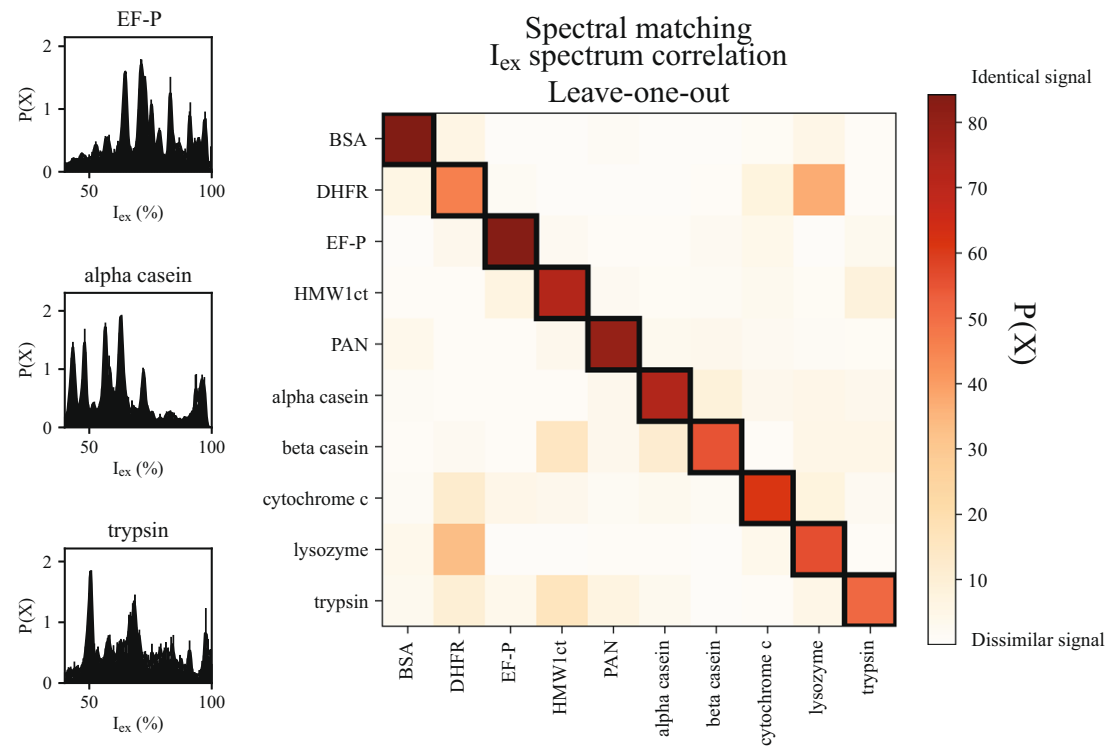

Fig. 3 Protein identification using nanopore spectrometry. a Baseline-corrected excluded current spectra of 9 tryptic digested proteins. Based on 3 individual measurements. $\mathbf{b}$ Leave-one-out spectral matching of the baseline corrected excluded current spectra using Euclidean cosine cross-correlation. With on the $\mathrm{Y}$-axis the protein that is being matched using leave-one-out matching with the proteins on the $\mathrm{X}$-axis. The black boxes represent the matches with the highest score. All nanopore measurements were performed in $1 \mathrm{M} \mathrm{KCl}$ buffered to $\mathrm{pH} 3.8$ using $50 \mathrm{mM}$ citric acid titrated with bis-tris-propane under an applied potential of $-70 \mathrm{mV}$. Recording was performed at $50 \mathrm{kHz}$ using an analog Bessel-filter at $10 \mathrm{kHz}$ and a digital Gaussian filter of $5 \mathrm{kHz}$. 
proteins are modified, heterogeneous proteins or low abundance proteins is challenging. Furthermore, mass spectrometers, are expensive and complicated devices that have high operational costs, which is why in protein analysis they are most often operated in centralized facilities ${ }^{2,3,6}$. In this work we introduce a method for protein analysis based on nanopores. Unlike MS, the electrical output signal arising from a nanopore can be easily interfaced with low-cost and portable devices. Nanopores have additional advantages as they detect single-molecules and they are amenable to incorporation into arrays of thousands of nanopores for high throughput analysis. However, despite nanopores having been applied successfully to sequence DNA, their use in protein identification has yet to be proven.

In this contribution, we describe a nanopore approach for the identification of proteins. Using FraC nanopores, we show that the tryptic digest of proteins generates clustered events from individual trypsinated peptides. Calibration using synthetic peptides corresponding to the expected complete hydrolysis of Gallus-gallus lysozyme (mass between 500 and $1700 \mathrm{Da}$ ) revealed that the nanopore captured all peptides and that an approximate relationship between the signal and the size of the peptide can be established (Fig. 2). Comparison between ten protein substrates showed a reasonably good correlation with MS analysis (Fig. 2 and Supplementary Fig. 5), and revealed that proteins can be recognized by spectral matching, which is similar to peptide mass fingerprinting (PMF), a technique used in early protein analysis. Although the proteins tested here are very different from each other, previous work showed that nanopore currents can detect differences in one single amino acid $20,38,39,42$ or single posttranslational modifications ${ }^{30,47}$. At present, we observe misassignments between peptide masses, which complicates the identification of unknown peptides and makes the recognition of unknown proteins challenging. However, nanopore currents report the volume of a peptide rather than its mass. Hence, we expect large improvements once calibration curves that take into consideration the exact volume of the peptide in solution will be used. We also found that peptide abundance measured by the nanopore matched relatively well that predicted by MS. Further research on the relationship between the capture frequency and the physico-chemical properties of the peptides will reveal the strength and limitation of nanopore analysis for quantification of peptides.

At the moment, the resolution of nanopore analysis does not yet match that of mass spectrometry. However, improvements can be envisaged. For example, upstream separation devices could be used, the interaction between the nanopore and the analytes can be improved, or nanopores with different sizes, shapes and physical properties can be used. Nonetheless, nanopores have distinguishable advantages: they can be integrated into inexpensive and portable devices ${ }^{1,3}$, they work in solution and they can be directly interfaced with other analytical devices such as liquid chromatography or spectroscopy devices. Further, nanopores measure a fundamentally different property of peptides compared to MS (volume rather than mass/charge). Hence, nanopores might be used alongside MS for the identification and/or quantification of analytes (e.g. isobaric peptides) that are not easily studied by MS. Furthermore, arrays of thousands of nanopores are currently under development in commercial devices, suggesting that nanopore arrays have the potential to allow highthroughput protein analysis. Finally, nanopores are singlemolecule sensors. Therefore, if a protease will be coupled to a nanopore directly ${ }^{34}$, this approach might be used for fingerprinting-based identification of single molecules. The latter might find application in the characterization of low abundance proteins or the heterogeneity of protein samples.

\section{Methods}

Chemicals. Potassium chloride, sodium chloride, urea, imidazole, $N, N$-Dimethyldodecylamine $N$-oxide (LDAO), n-Dodecyl $\beta$-D-maltoside (DDM), and lysozyme were received from Carl-Roth. 2-Amino-2-(hydroxymethyl)-1,3-propanediol (Tris) was obtained from Roche; citric acid, bovine serum albumin (BSA), and n-hexadecane were purchased from Acros. Pentane, magnesium chloride, dithiothreitol, iodoacetamide, trypsin, beta-casein, cytochrome C, alpha-casein, sheep blood and peptides were purchased from Sigma-Aldrich (Merck), ethanol from (Boomlab). 1,2-diphytanoyl-sn-glycero-3-phosphocholine (DPhPC), and sphingomyelin were received from Avanti Polar Lipids. Ni-NTA beads were obtained from Qiagen.

Protein digestion. In total $100 \mu \mathrm{g}$ of protein stock was taken and the volume was adjusted to $50 \mu \mathrm{l}$ using $20 \mathrm{mM}$ Tris. $\mathrm{HCl}$ buffer ( $\mathrm{pH} \mathrm{7.5).} \mathrm{A} \mathrm{final} \mathrm{concentration} \mathrm{of}$ $20 \mathrm{mM}$ dithiothreitol (DTT) was added to reduce any disulfide bonds. The sample was incubated at $37^{\circ} \mathrm{C}$ for $15 \mathrm{~min}$ followed by a denaturing step at $95^{\circ} \mathrm{C}$ for $15 \mathrm{~min}$. Afterwards, a $20 \mathrm{mM}$ iodoacetamide (IAA) was added and the sample was left to incubate for $15 \mathrm{~min}$ at room temperature in the dark in order to alkylate the reduced cysteine residues. Finally, the total volume was adjusted to $100 \mu \mathrm{l}$ using $100 \mathrm{mM}$ Tris Buffer ( $\mathrm{pH}$ 8.5).

Tryptic digestion was performed using a kit purchased from Sigma-Aldrich, containing proteomics grade trypsin singles. A total of $50 \mu \mathrm{l}$ of sample (containing $50 \mu \mathrm{g}$ of protein) was added to $1 \mu \mathrm{g}$ of mass-spec grade trypsin (1:50 enzyme:protein ratio) and the sample was subsequently incubated overnight at $37^{\circ} \mathrm{C}$. Since large $(>>2000 \mathrm{Da})$ peptides could clog the nanopore, the protein solution was passed through a centrifugal filter with a molecular weight cut-off of $3000 \mathrm{Da}$ (Amicon). Filtered samples were stored at $-20^{\circ} \mathrm{C}$ prior to use.

Fragaceatoxin C monomer purification. Fragaceatoxin $\mathrm{C}$ nanopores were expressed and purified as described previously ${ }^{26}$. In brief, a pT7-SC1 plasmid containing the G13F-FraC gene (Supplementary Table 1), was electrochemically transformed into BL21(DE3) competent Escherichia coli cells and grown overnight at $37^{\circ} \mathrm{C}$ on $\mathrm{LB}$ agar plates supplemented with $100 \mathrm{mg} / \mathrm{L}$ ampicillin and $1 \%$ glucose. On the next day, grown LB plates were solubilized into $200 \mathrm{~mL} 2 \mathrm{xYT}$ medium, supplemented with $100 \mathrm{mg} / \mathrm{L}$ ampicillin. Cultures were grown under constant shaking at $37^{\circ} \mathrm{C}$ until an optical density $\left(\mathrm{OD}_{600}\right)$ of 0.6 was reached. Afterwards, $0.5 \mathrm{mM}$ isopropyl $\beta$-D-1-thiogalactopyranoside was added for induction and growth continued overnight at $21^{\circ} \mathrm{C}$. Bacterial cells were pelleted using centrifugation $\left(6,000 \mathrm{~g}, 20 \mathrm{~min}, 4^{\circ} \mathrm{C}\right)$ and stored for at least one hour at $-80^{\circ} \mathrm{C}$. $100 \mathrm{~mL}$ (original culture) cell pellets were resuspended using $20 \mathrm{ml}$ lysis buffer, consisting of $150 \mathrm{mM} \mathrm{NaCl}$ buffered at $\mathrm{pH} 7.5$ using $15 \mathrm{mM}$ Tris base and supplemented with $1 \mathrm{mM} \mathrm{MgCl}$, $2 \mathrm{M}$ Urea, $0.2 \mathrm{mg} / \mathrm{mL}$ lysozyme, 0.2 units/mL DNase and $20 \mathrm{mM}$ imidazole. Solubilised pellets were vigorously shaken for 1 hour at room temperature. The lysate was disrupted fully by sonification for $60 \mathrm{~s}$ (duty cycle $30 \%$, output control 3) using a Branson Sonifier 450 . The lysate was centrifuged at $6000 \mathrm{~g}$ for $20 \mathrm{~min}$ at $4^{\circ} \mathrm{C}$ and the supernatant was transferred to a fresh $50 \mathrm{~mL}$ falcon tube. Subsequently, $200 \mu \mathrm{L}$ pre-washed Ni-NTA beads (Qiagen, stored at $4{ }^{\circ} \mathrm{C}$ ) was added and the falcon tube was incubated for $1 \mathrm{~h}$ at room temperature $\left(21^{\circ} \mathrm{C}\right)$ under constant rotation. The supernatant, incubated with beads, was added to a pre-washed Micro Bio-Spin column (Bio-Rad) and, afterwards, washed extensively using a buffer consisting of $150 \mathrm{mM} \mathrm{NaCl}$ buffered at pH 7.5 using $15 \mathrm{mM}$ Tris base supplemented with $20 \mathrm{mM}$ imidazole. The column was dried by centrifugation $(500 \mathrm{~g}, 1 \mathrm{~min})$ in order to remove residual wash buffer. Finally, monomeric protein was eluted $(500 \mathrm{~g}, 2 \mathrm{~min})$ after a $10 \mathrm{~min}$ incubation (room temperature, $21^{\circ} \mathrm{C}$ ) with $150 \mu \mathrm{L}$ of $150 \mathrm{mM} \mathrm{NaCl}$ buffered at $\mathrm{pH} 7.5$ using $15 \mathrm{mM}$ Tris base and supplemented with $300 \mathrm{mM}$ imidazole.

Sphingomyelin-DPhPC liposomes preparation. An equal mixture of $25 \mathrm{mg} 1,2$ diphytanoyl-sn-glycero-3-phosphocholine (DPhPC) and $25 \mathrm{mg}$ sphingomyelin (Brain, Porcine) was dissolved in $4 \mathrm{~mL}$ pentane containing $0.5 \mathrm{v} / \mathrm{v} \%$ ethanol. A film was formed on the side of a round bottom flask by application of heat under constant rotation, evaporating all solvents. The resulting film was dissolved in $10 \mathrm{~mL}$ of $150 \mathrm{mM} \mathrm{NaCl}$, buffered at $\mathrm{pH} 7.5$ using $15 \mathrm{mM}$ Tris base. The resulting liposome solution $(5 \mathrm{mg} / \mathrm{mL})$ was frozen $\left(-20^{\circ} \mathrm{C}\right)$ and thawed multiple times.

Fragaceatoxin C oligomerisation. Freeze-thawed liposomes were added to purified monomers of fragaceatoxin $\mathrm{C}$ in a mass ratio of 10:1 (liposomes:protein). Liposomes were left to incubate for $30 \mathrm{~min}$, at $37^{\circ} \mathrm{C}$, and later solubilised by the addition of $0.6 \mathrm{v} / \mathrm{v} \%$ LDAO. Subsequently, the solution was diluted 20 times with $150 \mathrm{mM} \mathrm{NaCl}$ buffered at pH 7.5 using $15 \mathrm{mM}$ Tris supplemented with $0.02 \%$ DDM. $200 \mu \mathrm{L}$ washed regenerated Ni-NTA were added and incubated for $1 \mathrm{~h}$ at room temperature $\left(21^{\circ} \mathrm{C}\right)$ under constant rotation.

Incubated Ni-NTA beads were transferred onto a pre-washed Micro Bio-Spin column (Bio-Rad) and washed extensively using a buffer consisting of $150 \mathrm{mM}$ $\mathrm{NaCl}$ buffered at $\mathrm{pH} 7.5$ using $15 \mathrm{mM}$ Tris base supplemented with $20 \mathrm{mM}$ imidazole and $0.02 \mathrm{v} / \mathrm{v} \%$ DDM. The Micro Bio-Spin column was dried by centrifugation $(500 \mathrm{~g}, 1 \mathrm{~min})$ in order to remove residual wash buffer. Protein was eluted $\left(500 \mathrm{~g}, 2 \mathrm{~min}\right.$ ) after a $10 \mathrm{~min}$ incubation (room temperature, $21^{\circ} \mathrm{C}$ ) with 
Event and $\mathrm{I}_{\mathbf{e x}} \%$ extraction. Peptide translocation events were extracted from the data using a threshold-search algorithm and characterized using a generalized flattop normal distribution function (gNDF).

We use a threshold function to determine all events at $3 \sigma$ from the baseline, and subsequently fit a generalized flat-top normal distribution. This distribution is a good descriptor for the events we observe, as it resembles a spike-like profile when $\beta<1$, a Gaussian profile when $\beta=1$, and a flat-top shape when $\beta>1$. We filter events where $\beta<1$, as these resemble a spike and the residual current (height) cannot be accurately estimated. Therefore, the events that we use to construct the residual current spectrum are all Gaussian or flat-top shaped, resulting in less variance between spectra.

This approach shares similarities with other methods to characterize events, such as MOSAIC ${ }^{50,51}$. In both cases, an idealized event are represented and a filter effect is applied. Advantageously, the gNDF describes both the filter effect and flattop profile in a closed form. This allows the discrimination based on the event shape, however, it is slower for event recognition than using the algorithms implemented in MOSAIC

$$
f(x)=\Delta I_{B}{ }^{*} \exp \left(-\left(\frac{(x-\mu)^{2}}{2 \sigma^{2}}\right)^{\beta}\right)+I_{O} \text { for } \beta>0
$$

where $\mu$ is the events center in the time domain with variance $\sigma^{2}$ and $\Delta \mathrm{I}_{\mathrm{B}}$ is the current difference $(\mathrm{pA})$ between the baseline $\left(\mathrm{I}_{\mathrm{O}}\right)$ and the event maximum. The variable $\beta$ describes the shape of the function.

We utilize the generalized flat-top normal distribution, which is also used for the estimation of the dwell time. We estimate the events based on the full-width at half maximum of this distribution. Therefore, the dwell time is not skewed in short events. We discard spike-like events with a $\beta<1$, however, we would not be able to determine the dwell time of these events in either algorithm.

$$
F W H M=2 \sigma \sqrt{2 \sqrt[\beta]{\ln 2}}
$$

where $\sigma$ equals the square root of the variance $\left(\sigma^{2}\right)$ and $\beta$ describes the shape parameter.

For each event, the excluded current was calculated by dividing the median current difference between the blockade $\left(\mathrm{I}_{\mathrm{B}}\right)$ and the median open pore current $\left(\mathrm{I}_{\mathrm{O}}\right)$ by $\mathrm{I}_{\mathrm{o}}$.

$$
I_{e x} \%=\frac{I_{B}-I_{o}}{I_{o}} * 100 \%=\frac{\Delta I_{B}}{I_{o}} * 100 \%
$$

Where $I_{B}$ is the blocked pore current, $I_{o}$ represents the open pore current, and $I_{e x} \%$ is the excluded current.

$I_{\text {ex }} \%$ spectrum construction and re-alignment. For each event, the $I_{\text {ex }} \%$ was calculated and a histogram between 0 and $100 \mathrm{I}_{\mathrm{ex}} \%$ was constructed with a binwidth of $1 \mathrm{I}_{\mathrm{ex}} \%$. The resulting histogram is called the excluded current spectrum, or abbreviated as the $\mathrm{I}_{\mathrm{ex}} \%$ spectrum.

In order to correct for shifting in the residual current due to experimental fluctuations (e.g. slight differences in salt concentration, temperature, and instrument offset), we performed spectral re-alignment. We selected one spectrum (per protein) as a reference to which we re-align. Then, we subtracted bin-by-bin each additional spectrum to the reference spectrum and collected the residual sum of squares (as "error"). The x-axis of each bin of the new spectrum was moved in steps of $0.05 \mathrm{I}_{\mathrm{ex}} \%$ and the error recorded. After performing 100 step-wise additions (total $+5 \mathrm{I}_{\mathrm{ex}} \%$ ) and 100 step-wise subtractions (total $-5 \mathrm{I}_{\mathrm{ex}} \%$ ) a plot of $\Delta \mathrm{I}_{\mathrm{ex}} \%$ versus the error was obtained showing the spectral offset where the error is minimal (Supplementary Fig. 3).

Data analysis. All the data were analysed using Python 3.7 and is contained within a Jupyter notebook as an ad-hoc script and is available from the corresponding authors upon reasonable request and at https://doi.org/10.5281/zenodo.5205565.

Mapping mass to excluded current. When the average $\mathrm{I}_{\mathrm{ex}} \%$ is plotted versus the peptide mass, we obtain a non-linear relationship, instead of a linear curve that would be expected if the excluded current would only depend on the mass of the peptide. It is important to notice that the observed current is reduced because of the steric exclusion of ions induced by the analyte. Steric exclusion correlates to the mass of a peptide due to the sum of partial volume ${ }^{52}$, and assumes no 3-dimensional structure, nor an interaction with the nanopore. We reasoned that the correlation between the molecular weight and $\mathrm{I}_{\mathrm{ex}} \%$ of peptides can be represented as a second order polynomial (Eq. (4) ${ }^{29}$. We have shown this relationship in a previous contribution, and also include the origin in the fit. We used the polyld function as implemented in the Numpy library to find the ideal fit around the data. We found the following parameters: $b_{2}=-1.33 \times 10^{-5}, b_{1}=7.23 \times 10^{-2}$, and $\mathrm{b}_{0}=3.28$.

$$
I_{\text {ex }, M S}(m)=b_{0}+b_{1} * m+b_{2} * m^{2}
$$

where $\mathrm{b}_{0}, \mathrm{~b}_{1}$, and $\mathrm{b}_{2}$ are the exponential terms. The input variable $m$ represents the mass of the peptide.
$\mathbf{I}_{\text {ex }} \%$ spectrum density from MS analysis. The mass of each peptide was converted to $\mathrm{I}_{\text {ex } \mathrm{MS}} \%$ using the fit of Fig. $2 \mathrm{c}$. Each value was then used as the center $(u)$ a peak. The spread of each peak was made using an arbitrary standard deviation $(\sigma)$ of $0.5 \mathrm{I}_{\mathrm{ex}} \%$. The amplitude ( $a$ ) of the peaks was matched to the area observed from ESI-MS measurements. Throughout this contribution, we utilized a ESI-MS containing an Orbitrap detector. The intensity of each ion is therefore measured as the amplitude of the free induction decay, which has a square relationship with the number of ions detected. Therefore, we had to take the square root of the intensity resulting in Eq. (5).

$$
g(x)=\sqrt{\sum_{i=0}^{n} a_{i}^{*} \exp \left(-\frac{\left(x-\hat{I}_{e x}\left(m_{i}\right)\right)^{2}}{2 \sigma^{2}}\right)}
$$

where $a_{i}$ is the area resulting from ESI-MS. $m_{i}$ is the mass of the peptide and is mapped to the excluded current using Eq. (4). $\sigma$ is the peak width.

Spectral matching. We analyzed the $\mathrm{I}_{\mathrm{ex}} \%$ spectra using a spectral matching algorithm incorporating the squared first derivate Euclidean cosine correlation (DEuc) (Eq. (6); which is advantageous, as it corrects for baseline sloping ${ }^{53}$. The DEuc is a direct result of the dot product equation and allows the estimation of the angle between two vectors, which can be used as a measure of similarity. We use the normalized counts of the excluded current spectra which are represented as vectors, e.g. $\overrightarrow{\boldsymbol{A}}=\left[a_{1}, a_{2}, \ldots, a_{n}\right]$ and $\overrightarrow{\boldsymbol{B}}=\left[b_{1}, b_{2}, \ldots, b_{n}\right]$. We estimate the derivative of $\vec{A}$ and $\vec{B}$ by numerical differentiation, resulting in $\Delta \vec{A}$ and $\Delta \vec{B}$. Usage of the derivative is advantageous as this is less sensitive to background noise, which is usually stochastically distributed.

$$
D E u c=\cos (\theta)=\frac{\Delta \vec{A} \cdot \Delta \vec{B}}{\|\Delta \vec{A}\|\|\Delta \vec{B}\|}=\frac{\left(\sum_{i=0}^{N} \Delta a_{i} \Delta b_{i}\right)^{2}}{\sum_{i=0}^{N} \Delta a_{i}^{2} * \sum_{i=0}^{N} \Delta b_{i}^{2}}
$$

We chose to only consider the $\mathrm{I}_{\mathrm{ex}} \%$ spectrum between 50 and $98 \mathrm{I}_{\mathrm{ex}} \%$, as noise below the limit-of-detection and fully blocked events may have skewed the comparison. Subsequently, we performed a leave-one-out comparison using the DEuc as a score, normalised to $100 \%$ for visualisation. The leave-one-out comparison compares the $\mathrm{I}_{\mathrm{ex}} \%$ spectrum of each measurement (sample), with the average $\mathrm{I}_{\mathrm{ex}} \%$ spectrum of each protein (database). For each comparison, we constructed a database that contains all measurements except for the sample. The score represented in Fig. $3 \mathrm{~b}$ of the main text are the average scores over all samples for each protein.

Reporting summary. Further information on research design is available in the Nature Research Reporting Summary linked to this article.

\section{Data availability}

All data and corresponding analysis generated in this study have been deposited in the Zenodo database under https://doi.org/10.5281/zenodo.5205565. Source data underlying Fig. 2b, $c$ and Supplementary Fig. 1 are provided as a Source Data file. Source data are provided with this paper.

\section{Code availability}

All custom code and algorithms used in this study are available at https://doi.org/ 10.5281/zenodo.5205565.

Received: 23 February 2021; Accepted: 2 September 2021; Published online: 04 October 2021

\section{References}

1. Baaken, G., Ankri, N., Schuler, A.-K., Rühe, J. \& Behrends, J. C. Nanoporebased single-molecule mass spectrometry on a lipid membrane microarray. ACS Nano 5, 8080-8088, https://doi.org/10.1021/nn202670z (2011).

2. Suhre, K., McCarthy, M. I. \& Schwenk, J. M. Genetics meets proteomics: perspectives for large population-based studies. Nat. Rev. Genet. https:// doi.org/10.1038/s41576-020-0268-2 (2020).

3. Timp, W. \& Timp, G. Beyond mass spectrometry, the next step in proteomics. Sci. Adv. 6, eaax8978, https://doi.org/10.1126/sciadv.aax8978 (2020).

4. Aebersold, R. \& Mann, M. Mass spectrometry-based proteomics. Nature 422, 198-207, https://doi.org/10.1038/nature01511 (2003).

5. Aebersold, R. \& Mann, M. Mass-spectrometric exploration of proteome structure and function. Nature 537, 347-355, https://doi.org/10.1038/ nature19949 (2016)

6. Armah, S., Ferruzzi, M. G. \& Gletsu-Miller, N. Feasibility of massspectrometry to lower cost and blood volume requirements for assessment of 
B vitamins in patients undergoing bariatric surgery. Metabolites 10, 240, https://doi.org/10.3390/metabo10060240 (2020).

7. Soskine, M. et al. An engineered ClyA nanopore detects folded target proteins by selective external association and pore entry. Nano Lett. 12, 4895-4900, https://doi.org/10.1021/nl3024438 (2012).

8. Stefureac, R., Waldner, L., Howard, P. \& Lee, J. S. Nanopore analysis of a small 86-residue protein. Small 4, 59-63, https://doi.org/10.1002/smll.200700402 (2008).

9. Si, W. \& Aksimentiev, A. Nanopore sensing of protein folding. ACS Nano 11, 7091-7100, https://doi.org/10.1021/acsnano.7b02718 (2017).

10. Nivala, J., Mulroney, L., Li, G., Schreiber, J. \& Akeson, M. Discrimination among protein variants using an unfoldase-coupled nanopore. ACS Nano 8 , 12365-12375, https://doi.org/10.1021/nn5049987 (2014).

11. Huo, M.-Z., Li, M.-Y., Ying, Y.-L. \& Long, Y.-T. Is the Volume Exclusion Model Practicable for Nanopore Protein Sequencing? Anal. Chem. 93, 11364-11369, https://doi.org/10.1021/acs.analchem.1c00851 (2021).

12. Zernia, S., van der Heide, N. J., Galenkamp, N. S., Gouridis, G. \& Maglia, G. Current blockades of proteins inside nanopores for real-time metabolome analysis. ACS Nano 14, 2296-2307, https://doi.org/10.1021/acsnano.9b09434 (2020).

13. Willems, K. et al. Engineering and modeling the electrophoretic trapping of a single protein inside a nanopore. ACS Nano 13, 9980-9992, https://doi.org/ 10.1021/acsnano.8b09137 (2019).

14. Cao, C. et al. Discrimination of oligonucleotides of different lengths with a wild-type aerolysin nanopore. Nat. Nanotechnol. 11, 713-718, https://doi.org/ 10.1038/nnano.2016.66 (2016).

15. Baaken, G. et al. High-resolution size-discrimination of single nonionic synthetic polymers with a highly charged biological nanopore. ACS Nano 9 , 6443-6449, https://doi.org/10.1021/acsnano.5b02096 (2015).

16. Lucas, F. L. R. et al. The manipulation of the internal hydrophobicity of FraC nanopores augments peptide capture and recognition. ACS Nano https:// doi.org/10.1021/acsnano.0c09958 (2021).

17. Sutherland, T. C. et al. Structure of peptides investigated by nanopore analysis. Nano Lett. 4, 1273-1277, https://doi.org/10.1021/nl049413e (2004).

18. Robertson, J. W. F. \& Reiner, J. E. The utility of nanopore technology for protein and peptide sensing. Proteomics 18, 1-36, https://doi.org/10.1002/ pmic.201800026 (2018)

19. Robertson, J. W. F. et al. Single-molecule mass spectrometry in solution using a solitary nanopore. Proc. Natl Acad. Sci. USA 104, 8207-8211, https://doi.org/ 10.1073/pnas.0611085104 (2007)

20. Restrepo-Pérez, L. et al. Resolving chemical modifications to a single amino acid within a peptide using a biological nanopore. ACS Nano 13, 13668-13676, https://doi.org/10.1021/acsnano.9b05156 (2019).

21. Reiner, J. E., Kasianowicz, J. J., Nablo, B. J. \& Robertson, J. W. Theory for polymer analysis using nanopore-based single-molecule mass spectrometry. Proc. Natl Acad. Sci. USA 107, 12080-12085, https://doi.org/10.1073/ pnas.1002194107 (2010).

22. Li, Z., He, B. \& Feng, W. Evaluation of bottom-up and top-down mass spectrum identifications with different customized protein sequences databases. Bioinformatics 36, 1030-1036, https://doi.org/10.1093/ bioinformatics/btz733 (2020).

23. Huang, G., Willems, K., Soskine, M., Wloka, C. \& Maglia, G. Electro-osmotic capture and ionic discrimination of peptide and protein biomarkers with FraC nanopores. Nat. Commun. 8, 935, https://doi.org/10.1038/s41467-017-01006-4 (2017).

24. Gu, L. Q., Braha, O., Conlan, S., Cheley, S. \& Bayley, H. Stochastic sensing of organic analytes by a pore-forming protein containing a molecular adapter. Nature 398, 686-690, https://doi.org/10.1038/19491 (1999).

25. Mohammad, M. M. M. \& Movileanu, L. Excursion of a single polypeptide into a protein pore: simple physics, but complicated biology. Eur. Biophys. J. 37, 913-925, https://doi.org/10.1007/s00249-008-0309-9 (2008).

26. Mutter, N. L. et al. Preparation of Fragaceatoxin C (FraC) Nanopores; 2021; pp 3-10. https://doi.org/10.1007/978-1-0716-0806-7_1.

27. Movileanu, L., Howorka, S., Braha, O. \& Bayley, H. Detecting protein analytes that modulate transmembrane movement of a polymer chain within a single protein pore. Nat. Biotechnol. 18, 1091-1095, https://doi.org/10.1038/80295 (2000).

28. Kasianowicz, J. J., Brandin, E., Branton, D. \& Deamer, D. W. Characterization of individual polynucleotide molecules using a membrane channel. Proc. Natl Acad. Sci. USA 93, 13770-13773, https://doi.org/10.1073/pnas.93.24.13770 (1996).

29. Huang, G., Voet, A. \& Maglia, G. FraC nanopores with adjustable diameter identify the mass of opposite-charge peptides with 44 Dalton resolution. Nat. Commun. 10, 835, https://doi.org/10.1038/s41467-019-08761-6 (2019).

30. Restrepo-Pérez, L., Wong, C. H., Maglia, G., Dekker, C. \& Joo, C. Label-free detection of post-translational modifications with a nanopore. Nano Lett. 19, 7957-7964, https://doi.org/10.1021/acs.nanolett.9b03134 (2019).
31. James, P., Quadroni, M., Carafoli, E. \& Gonnet, G. Protein identification by mass profile fingerprinting. Biochem. Biophys. Res. Commun. 195, 58-64, https://doi.org/10.1006/bbrc.1993.2009 (1993).

32. Pappin, D. J. C., Hojrup, P. \& Bleasby, A. J. Rapid identification of proteins by peptide-mass fingerprinting. Curr. Biol. 3, 327-332, https://doi.org/10.1016/ 0960-9822(93)90195-T (1993).

33. Cottrell, J. S. \& Sutton, C. W. The identification of electrophoretically separated proteins by peptide mass fingerprinting. In Protein and Peptide Analysis by Mass Spectrometry; Humana Press: New Jersey; pp. 67-82. https://doi.org/10.1385/0-89603-345-7:67.

34. Zhang, S. et al. Bottom-up Fabrication of a Multi-Component Nanopore Sensor That Unfolds, Processes and Recognizes Single Proteins. bioRxiv 2020, 2020.12.04.411884. https://doi.org/10.1101/2020.12.04.411884.

35. Chinappi, M., Yamaji, M., Kawano, R. \& Cecconi, F. Analytical model for particle capture in nanopores elucidates competition among electrophoresis, electroosmosis, and dielectrophoresis. ACS Nano 14, 15816-15828, https:// doi.org/10.1021/acsnano.0c06981 (2020).

36. Li, S., Cao, C., Yang, J. \& Long, Y.-T. Detection of peptides with different charges and lengths by using the aerolysin nanopore. ChemElectroChem 6 , 126-129, https://doi.org/10.1002/celc.201800288 (2019).

37. Angevine, C. E., Robertson, J. W. F., Dass, A. \& Reiner, J. E. Laser-based temperature control to study the roles of entropy and enthalpy in polymernanopore interactions. Sci. Adv. 7, eabf5462, https://doi.org/10.1126/ sciadv.abf5462 (2021).

38. Chavis, A. E. et al. Single molecule nanopore spectrometry for peptide detection. ACS Sens. 2, 1319-1328, https://doi.org/10.1021/acssensors.7b00362 (2017).

39. Asandei, A., Rossini, A. E., Chinappi, M., Park, Y. \& Luchian, T. Protein nanopore-based discrimination between selected neutral amino acids from polypeptides. Langmuir 33, 14451-14459, https://doi.org/10.1021/ acs.langmuir.7b03163 (2017).

40. Wolfe, A. J., Mohammad, M. M., Cheley, S., Bayley, H. \& Movileanu, L. Catalyzing the translocation of polypeptides through attractive interactions. J. Am. Chem. Soc. 129, 14034-14041, https://doi.org/10.1021/Ja0749340 (2007).

41. Cao, C. et al. Single-molecule sensing of peptides and nucleic acids by engineered aerolysin nanopores. Nat. Commun. 10, 4918, https://doi.org/ 10.1038/s41467-019-12690-9 (2019).

42. Ouldali, H. et al. Electrical recognition of the twenty proteinogenic amino acids using an aerolysin nanopore. Nat. Biotechnol. 38, 176-181, https:// doi.org/10.1038/s41587-019-0345-2 (2020).

43. Yakovlieva, L. et al. A $\beta$-hairpin epitope as novel structural requirement for protein arginine rhamnosylation. Chem. Sci. 2021. https://doi.org/10.1039/ D0SC05823H.

44. Galenkamp, N. S., Biesemans, A. \& Maglia, G. Directional conformer exchange in dihydrofolate reductase revealed by single-molecule nanopore recordings. Nat. Chem. 12, 481-488, https://doi.org/10.1038/s41557-0200437-0 (2020)

45. Walvoort, M. T. C. C. et al. Antibodies from multiple sclerosis patients preferentially recognize hyperglucosylated adhesin of non-typeable haemophilus influenzae. Sci. Rep. 6, 39430, https://doi.org/10.1038/srep39430 (2016).

46. Sinha, A. \& Mann, M. A Beginner's guide to mass spectrometry-based. Proteom. Biochem. (Lond.). 42, 64-69, https://doi.org/10.1042/BIO20200057 (2020).

47. Rosen, C. B., Rodriguez-Larrea, D. \& Bayley, H. Single-molecule site-specific detection of protein phosphorylation with a nanopore. Nat. Biotechnol. 32, 179-181, https://doi.org/10.1038/nbt.2799 (2014).

48. Cheley, S. et al. Spontaneous oligomerization of a Staphylococcal alphahemolysin conformationally constrained by removal of residues that form the transmembrane beta-barrel. Protein Eng. Des. Sel. 10, 1433-1443, https:// doi.org/10.1093/protein/10.12.1433 (1997).

49. Walvoort, M. T. C. et al. Erratum: antibodies from multiple sclerosis patients preferentially recognize hyperglucosylated adhesin of non-typeable Haemophilus influenzae. Sci. Rep. 7, 44969, https://doi.org/10.1038/srep44969 (2017).

50. Forstater, J. H. et al. MOSAIC: a modular single-molecule analysis interface for decoding multistate nanopore data. Anal. Chem. 88, 11900-11907, https:// doi.org/10.1021/acs.analchem.6b03725 (2016).

51. Balijepalli, A. et al. Quantifying short-lived events in multistate ionic current measurements. ACS Nano 8, 1547-1553, https://doi.org/10.1021/nn405761y (2014).

52. Harpaz, Y., Gerstein, M. \& Chothia, C. Volume changes on protein folding. Structure 2, 641-649, https://doi.org/10.1016/S0969-2126(00)00065-4 (1994).

53. Li, J., Hibbert, D. B., Fuller, S. \& Vaughn, G. A comparative study of point-topoint algorithms for matching spectra. Chemom. Intell. Lab. Syst. 82, 50-58, https://doi.org/10.1016/j.chemolab.2005.05.015 (2006). 


\section{Acknowledgements}

This work is part of the research program of the Foundation for Fundamental Research on Matter (FOM), which is part of the Netherlands Organization for Scientific Research (NWO) under grant number 16SMPS05. We acknowledge financial support by an ERC consolidator grant (number: 726151 to G.M.), the Dutch Organization for Scientific Research (VENI 722.016.006), and the European Union through the Rosalind Franklin Fellowship COFUND project 60021 (both to M.T.C.W.).

\section{Author contributions}

F.L., R.V., L.Y., M.W., and G.M. designed the experiments. G.M. and M.W. supervised the project. F.L. and R.V. performed the experiments. F.L. and R.V. performed the data analysis. L.Y. and R.V. performed the MS-based analysis. F.L., R.V., and G.M. wrote the manuscript.

\section{Competing interests}

The authors declare no competing interests.

\section{Additional information}

Supplementary information The online version contains supplementary material available at https://doi.org/10.1038/s41467-021-26046-9.

Correspondence and requests for materials should be addressed to Giovanni Maglia.

Peer review information Nature Communications thanks the anonymous reviewers for their contribution to the peer review of this work.

Reprints and permission information is available at http://www.nature.com/reprints

Publisher's note Springer Nature remains neutral with regard to jurisdictional claims in published maps and institutional affiliations.

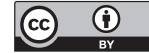
appropriate credit to the original author(s) and the source, provide a link to the Creative Commons license, and indicate if changes were made. The images or other third party material in this article are included in the article's Creative Commons license, unless indicated otherwise in a credit line to the material. If material is not included in the article's Creative Commons license and your intended use is not permitted by statutory regulation or exceeds the permitted use, you will need to obtain permission directly from the copyright holder. To view a copy of this license, visit http://creativecommons.org/ licenses/by/4.0/.

(C) The Author(s) 2021 